\title{
Bridging the Accessibility Gap in Open Educational Resources
}

\author{
Rosa Navarrete • Sergio Luján-Mora
}

Received: date / Accepted: date

\begin{abstract}
Open Educational Resources (OER) are being fostered as a global movement for providing educational opportunities to all. However, people with disabilities are still excluded from full participation because of the lack of accessibility of OER websites, as well as of the resources themselves. This work presents a proposal for the design of OER websites that would enable equitable access for all users. This design aims to bridge the accessibility gap through the personalization of the whole OER environment to facilitate an accessible User Experience (UX) based on a user profile that includes the self-identification of disability status. This profile configures not only the "look and feel" of the interface but also the delivery of educational resources suitable for this user profile. To achieve this purpose, the design goes beyond compliance with the ISO/IEC 40500 W3C Web Content Accessibility Guidelines (WCAG) 2.0 , since it includes the personalization of the accessible experience through usability considerations and adaptations of educational resources. The delivery of educational resources matches the user's profile with regards to their sensory abilities, cognitive faculties and their requirements of functionality control, display layout, and language. As a proof of concept, we have developed an OER website based on this design and have conducted a set of UX tests that include users with
\end{abstract}

\section{R. Navarrete,}

Escuela Politécnica Nacional, Departamento de Informática y Ciencias de la Computación, Quito-Ecuador

E-mail: rosa.navarrete@epn.edu.ec

S. Luján-Mora,

University of Alicante, Department of Software and Computing Systems, Alicante-Spain

E-mail: sergio.lujan@ua.es different disabilities. The test results confirm the feasibility and suitability of our design regarding accessible UX. Finally, the contribution of this document arises from the explicit recognition of the particular needs associated with the disability profiles to establish the response of the entire OER system which enables a truly inclusive experience by exempting the user from performing configuration tasks.

Keywords Open Educational Resources · accessible UX · accessibility · adaptations of educational resources · Access for All metadata

\section{Introduction}

The commitment to an inclusive and equitable quality education is one of the goals of the United $\mathrm{Na}$ tions 2030 Agenda for Sustainable Development [1]. This goal is supported by Education for All, an initiative of UNESCO [2], that highlights accessibility as a key aspect of its achievement.

In 2002, UNESCO adopted the term Open Educational Resources (OER) to refer to digital content available in open conditions for learning purposes [3]. OER include syllabi, lectures, textbooks, assessments, and any other learning materials published in distinct formats such as web pages, PDF documents, videos, podcasts, images and more. In order to be open, these educational resources must be released under an intellectual property license that permits their free use [4] and are usually stored in repositories available through websites.

The OER movement highlights the inclusive vision of educational opportunities offered through the openness of access to all people regardless of their particular requirements [5-7]. Nevertheless, OER websites are still 
not accessible because they have not been considered as a whole, i.e. their interface could be accessible to users though not educational resources and the other way around. Therefore, people with disabilities face barriers to access and interaction with both the OER websites and the educational resources provided by these websites [8-10].

In terms of web interaction, disabilities include loss of, or decreased or disordered sight, hearing or movement of the upper limbs, in addition to cognitive disabilities and problems related to aging [11]. The statistics of the World Health Organization report 285 million people worldwide with visual impairments [12] and 360 million people with a disabling hearing loss [13]. Furthermore, the population over 60 years of age will increase from 901 million in 2015 to nearly 2.1 billion in 2050 [14], which implies a significant growth in people with disabilities due to aging. These statistics make it possible to put into perspective the impact of the declaration of the United Nations [15] that recognizes that people with disabilities are still excluded from equitable access to education, employment, healthcare and social and legal support systems.

In the last years, there have been improvements with regard to web accessibility on learning contexts. For example, some OER websites such as OER Commons and MERLOT, have enhanced accessibility by including accessibility descriptors to tag the resources, and the Floe Project [16] has presented a customization of the web environment to improve its suitability for users.

Nevertheless, accessibility regarding OER websites has not been addressed with an integrative approach. For this reason, in this paper, we propose a design of OER websites that considers previous works and goes further. This design aims to bridge the accessibility gap in OER websites through the personalization of the entire OER environment to the particular needs of a wide range of users, especially users with disabilities. This personalization provides an accessible User Experience (UX) for users with disabilities that considers accessibility, usability and Information Architecture (IA) as the basic design principles of UX.

To enable personalization, this design is founded on the definition of a user profile that includes a disability specification and the particular preferences and requirements of the user, including the preferred language. The user profile modifies the presentation of the web interface by activating characteristics that facilitate the user's perception, comprehension, navigation and operation. In addition, the user profile controls the searching of resources to provide resources adapted to the user's particular method of perception and interaction.
Moreover, as a proof of concept, we have developed and implemented an OER website based on our design, which has been named OERfAll as an acronym for "Open Educational Resources for All". To prove the validity of our proposal, we have conducted a comparative evaluation of the OERfAll website with another well known OER website. This evaluation included accessibility tests with automatic tools and UX tests conducted by users who have different disabilities. The positive outcomes obtained for the OERfAll website enable us to argue that accessibility for users with disabilities has been enhanced through this design approach. So, it could be helpful for guiding new developments in distinct web environments.

The structure of the paper is as follows. Section 2 presents an overview of previous works related to this proposal. Section 3 addresses the current state of some important OER websites and our OER website regarding the accessibility design approach presented in this work. Section 4 describes the design aspects of OER websites based on the elements required for an accessible UX. Section 5 presents the OER website developed as a proof of concept by applying this proposal design; also, it includes some use cases. Section 6 presents an evaluation of OERfAll that includes an accessibility evaluation test and a set of users' testing involving users with different disabilities. Finally, in Section 7 the most relevant outcomes of this work are pointed out.

\section{Related Works}

Despite the fact that accessibility has been widely addressed in research works, there are very few works directly concerned with OER and accessibility; most of them are focused on accessibility evaluation, for instance $[8,9]$. However, there are a significant number of works related to the topics involved in our proposal. Some of them are mentioned next:

- Adaptations or personalization of web resources. The Floe Project is presented in [16]; this project sets out to support a perspective of accessibility based on the recognition of the diversity of users with and without disabilities and the customization of the environment to fulfill their needs. In [17] the development of web-based service based on the progressive adoption of personalization is proposed as a way to improve accessibility. Similarly, the benefits of a user-focused approach to the accessibility of web resources are highlighted in [18].

Furthermore, in $[19,20]$ the authors propose a set of tools to develop accessible and adaptable learning objects that would enable an accessible relationship 
to users' needs. These studies use IMS Access for All metadata for implementation. In [21], a proposal for a system that transforms a media file from one format to another to produce a personalized learning object based on the requirements of a user is presented.

- Accessibility conditions regarding specific disabilities. The development of educational resources accessible to deaf people is addressed in $[22,23]$.

The problems encountered by blind people in web content that go beyond the aspects covered by accessibility guidelines are presented in [24]. Further, in [25] the authors propose an improvement of the web browsing experience by adapting and personalizing the software to users' preferences and available devices to accommodate different levels of users' visual impairments.

The requirements of web-based learning materials for people with dyslexia are addressed in [26-30]. The problems of using pointing devices and the prevalence of using keyboard for people with physical or motor disabilities is presented in [31].

The accessibility issues for people with cognitive disabilities are presented in [32] and the issues concerning older people are addressed in [33].

- Issues related to metadata. The generation of IMS Access for All Metadata to describe learning objects through components embedded in authoring tools is described in $[34,35]$. Different approaches about metadata for OER are presented in [36,37].

- Accessibility and usability issues in web content. Both, accessibility and usability to improve content for educational context are addressed in $[10,38,39]$.

While the above-mentioned related works address the accessibility of elements that are part of an OER website, such as the learning objects $[19,20]$ or the website interface [16], in a separate way, the novelty of our work is the holistic approach to accessibility personalized according to users' disability profile to enable an accessible UX. In our approach, the system automatically adjusts accessibility features for particular users' needs according to each disability. Therefore, users are not required to face complex configuration tasks of the interface as some related works propose $[19,20]$.

The system response to users' disability profiles is produced through an adaptive interface that is complemented with the delivering of educational resources that meet access modality suitable for users according to their disability besides other requirements of visualization, operation, educational complexity, and language. Furthermore, the availability of educational resources with different access modes and accessibility features does not rely solely on original resources but also in the adaptations of original resources [21]. All resources and their adaptations are stored in the catalog of resources.

Moreover, we have developed and tested an OER website based on the proposed approach which enables us to argue about their functionality and relevance in accessibility enhancement. The aspects of the proposed design are presented in Section 4.

\section{Current state of OER Websites}

Our design approach for OER websites focuses explicitly on the accessibility for users with disabilities [40]. We have reviewed the current state of some well-known and prestigious OER websites regarding this accessibility approach. We aim to verify if these websites have implemented any feature of this design approach for accessibility. Table 1 presents the list of features that have been examined in some OER websites and in our OERfAll website. These features are explained in the following.

- User identification: this entails whether a user can be identified on the OER website; such identification can be conducted by a Login process or by preserving the user's identity through another mechanism (i.e. HTTP cookies).

- Define users' disability profile: this concerns the extend to which a user can state their disability profile to guide the fulfillment of their accessibility requirements in the whole OER website.

- Define accessibility options for searching: the extend to which the website has incorporated accessibility options as parameters for searching educational resources. These options could be explicitly selected by the user or defined automatically by the system based on the accessibility requirements of a disability profile selected by the user.

- Adaptive interface layout according to user disability: examining whether the interface has an adaptive behavior in response to user disability.

- Customize the interface layout: examining whether the website enables users the customization of the interface layout to enhance UX.

- Define interface language: this addresses the extend to which the user can define the language of the interface.

- Persistent accessibility options for searching: this regards whether the accessibility options required for user are preserved for future access to the OER website.

- Persistent customization (interface layout and language): this addresses whether the customization of 
Table 1: Comparison of features in OER websites

\begin{tabular}{|c|c|c|c|c|c|c|c|}
\hline \multirow{2}{*}{ Feature } & \multicolumn{7}{|c|}{ OER Websites } \\
\hline & $\begin{array}{l}\text { OER } \\
\text { Commons }\end{array}$ & MERLOT & $\begin{array}{l}\text { MIT } \\
\text { OCW }\end{array}$ & OLI & ARIADNE & OpenStax & OERfAll \\
\hline User identification & $\begin{array}{l}\text { Yes } \\
(\operatorname{login})\end{array}$ & $\begin{array}{l}\text { Yes } \\
(\operatorname{login})\end{array}$ & Not & $\begin{array}{l}\text { Yes } \\
(\operatorname{login})\end{array}$ & Not & Not & $\begin{array}{l}\text { Yes } \\
\text { (HTTP cookies) }\end{array}$ \\
\hline Define user disability profile & Not & Not & Not & Not & Not & Not & Yes \\
\hline $\begin{array}{l}\text { Define accessibility options } \\
\text { for searching }\end{array}$ & $\begin{array}{l}\text { Yes } \\
\text { (by user) }\end{array}$ & $\begin{array}{l}\text { Yes (limited) } \\
\text { (by user) }\end{array}$ & Not & Not & Not & Not & $\begin{array}{l}\text { Yes } \\
\text { (by profile) }\end{array}$ \\
\hline $\begin{array}{l}\text { Adaptive interface layout } \\
\text { according to users' disability }\end{array}$ & Not & Not & Not & Not & Not & Not & Yes \\
\hline Customize the interface layout & Yes & Not & Not & Not & Not & Not & Yes \\
\hline Define interface language & Not & Yes & Not & Not & Not & Not & Yes \\
\hline $\begin{array}{l}\text { Persistent accessibility options } \\
\text { for searching }\end{array}$ & Not & Not & Not & Not & Not & Not & Yes \\
\hline $\begin{array}{l}\text { Persistent customization } \\
\text { (interface layout and } \\
\text { language) }\end{array}$ & Not & Not & Not & Not & Not & Not & Yes \\
\hline $\begin{array}{l}\text { Deliver resources matching } \\
\text { accessibility requirements of users }\end{array}$ & Not & Not & Not & Not & Not & Not & Yes \\
\hline
\end{tabular}

the interface layout and interface language are preserved for future user access to the OER website.

- Deliver resources matching accessibility requirements for users: this is whether the OER website delivers educational resources according to the access mode suitable for the user.

It is worth noting some important issues of OER websites presented in Table 1. In MERLOT, accessibility information is considered as "limited" because it mostly refers to the accessibility policy of the organization that provides the online materials. For this reason, it is not a helpful tool for users with disabilities, because they can not select appropriate formats of educational resources based on this information. Although MERLOT website offers language customization, we have encountered some misinterpretation in translation. For example, "accessibility information form" is translated in the Spanish language as the equivalent of "access information form". Furthermore, the user profile in MERLOT is a member profile that describes affiliation, contact information and similar. Only the preferred language for searches is preserved for future access. In OER Commons, the user profile is related to a member profile, so it saves contact information, subjects of interest and similar. This profile does not preserve accessibility features defined by a user. These features need to be defined each time of access. Similarly, the choices in interface layout selected by a user are not preserved for future access.

As has been exposed, the integrative approach of our proposal implemented in the OERfAll website presents advantages about the current state of OER websites regarding the accessibility requirements of users with disabilities. In the rest of the paper, we present detailed information about OERfAll design that leads to the values displayed in Table 1 for this website.

\section{Design Issues}

From our perspective, a disability arises from a failed outcome in the interaction of a user with an OER environment. The interaction quality depends on the suitability of the OER environment to fit the abilities and preferences of a user.

Based on these premises, we propose that the design of an OER environment accessible for users must be based on the concept of accessible UX [41] that emphasizes the recognition and understanding of users' needs [42] as a paramount consideration for users with disabilities. This approach to design seeks to ensure an accessible UX by enhancing the suitability of the website to match the users' needs regarding their capabilities and goals [43].

UX comprises all aspects of users' interaction with a website aimed at the achievement of their goals [44]. Some authors agree that UX depends on the context of the website and the individual expectations of users [43]. In consequence, UX involves pragmatic aspects such as efficiency, perspicuity and dependability on websites, as well as hedonic and attractiveness aspects [45].

To improve the UX of users with disabilities we have considered three fundamental aspects for design: acces- 
sibility, usability, and IA [39]. The accessibility and usability as components of UX are tightly interwoven [46] and both issues need to be properly integrated to make the website accessible and usable $[47,48]$. Further, IA is critical for UX [46] because the use of efficient metadata enhances the searching of resources based on a common system of identification and provides a meaningful navigation menu of categories of resources based on their descriptors.

In addition to these components, this design incorporates the concept of the adaptations of educational resources to ensure the delivery of suitable resources according to a user profile.

In the following, each design component is explained, as well as their integration into the OER environment to support the accessible UX.

\subsection{Web accessibility}

The goal of web accessibility is to enable people with disabilities to use the web on equal terms with other users [49]. The accessibility approach considered in the proposed design is twofold. On the one hand, the design ensures compliance with the ISO/IEC 40500 W3C WCAG 2.0 standard [50] at level AA, which is also the level demanded in web accessibility legislation in many countries. For this reason, the homepage of our OERfAll website provides accessibility for users from their first interaction.

On the other hand, the proposed approach to accessibility prioritizes users' needs and goals in the OER website. Therefore, to bridge the accessibility gap, we have personalized the configuration of the OER website based on a user profile that includes self-identification of disability status and visualization preferences. While self-identification is voluntary, providing this information is critical to accurately offer the best experience to users. Based on the user profile, the website personalizes and enhances accessibility characteristics and ensures that the delivery of resources matches the user profile. For instance, if a user declares a Deafness profile, only the videos with subtitles could be offered; if a user declares a Simplify profile, educational resources with educational complexity simplified could be offered.

We have not considered compliance with WCAG 2.0 level AAA of the entire website because this practice is not advisable as a regular policy [50]. Instead, the level AAA Success Criteria are applied according to each disability profile focusing on improvements for specific users' needs. For example, the Success Criteria "2.4.8 Location: information about the users' location within a set of web pages" is available by applying the Gen- eral Technique G65: providing a breadcrumb trail [51] is associated with the Simplify profile.

\subsection{Web usability}

Web usability is a complex concept that involves multiple attributes. Our design has considered the terms represented in published definitions: ease of use, effectiveness (reliability and completeness), efficiency (the effort and time invested), and satisfaction [52], all concerning the achievement of users' goals [53].

This design has selected the guidelines more relevant to improving the usability of this type of website [39]. These guidelines come from the international standard ISO 9241-151:2008 Guidance on World Wide Web user interfaces [54] and the set of usability guidelines published by the U.S. Government Department of Health and Human Services [55]. The selected guidelines are focused on three aspects:

- Task oriented. The website should be oriented towards task achievement. The homepage should prioritize the purpose of the website by providing access to the resources. The search and advanced search of resources should be highlighted in the center of the page to facilitate the attainment of users' goals. The homepage should offer the selection of the user profile as well as the selection of advanced options of configuration.

- Enhancing search and retrieval of resources. The search of resources should consider not only the conventional parameters of searching (subject, educational level, and format) but also the perception abilities of users defined through their profile. The presentation of the search results should be configurable (number of results per page, pagination). The advanced search should enable search refinement based on simultaneous parameters.

- Customized environment. The environment of the website should be configurable through the features activated by the selection of a user profile and advanced features. The configuration could affect the "look and feel" of the interface (typefaces, colors, shapes and layout, as well as the behavioral of links and menus) besides the activation of "navigation aids" (e.g., breadcrumb menu).

\subsection{Information Architecture}

IA is based on the metadata of resources and involves information organization considering users and the context of use [56]. The metadata standard defines a set of 
values known as "controlled vocabulary", which enable interoperability and conformability with the standards.

OER websites require a metadata standard that provides a structure of defined elements or specific descriptors to describe an educational resource and enable the categorization of resources. Not all educational metadata standards include accessibility descriptors and, thus, they fail to identify educational resources for users with disabilities [37].

Therefore, in the proposed design we have considered two metadata standards: the IMS Access For All Specification (AfA) [57], adopted as the standard ISO/IEC 24751-2:2008 [58] and the Learning Object Metadata (LOM) standard [59]. AfA is a metadata standard for digital content that includes accessibility descriptors and LOM is a metadata standard used to identify the resources, their subject matter, their educational level, their contributor, and their format.

\subsection{Adaptations of educational resources}

The adaptability of OER can overcome accessibility issues by producing resources accessible for more people [60]. To achieve this goal, the structure of educational resources has been considered as a "puzzle" whereby each piece can be replaced by an alternative modality or adaptation that matches the access mode based on the perception capabilities of the user. The educational resources can be adapted, partially or wholly.

As a result, it is possible to obtain new versions of the same educational resource with an equivalent learning purpose, though involving different sensory perceptions. In particular, each version of the resource aims to comply with requirements provided by users with disabilities. Therefore, it is important to bear in mind that a resource could have more than one access mode because of the access mode of each of its components. Adaptations are intended to ensure that the adapted resource only has components of which access mode is suitable to the user, according to their disability profile.

Moreover, this approach to accessibility in OER websites and educational resources is aligned with the purpose of Universal Design for Learning [61], which claims that learning is impossible if the information is imperceptible to the learner.

Figure 1 shows a schematic representation of the adaptation of a resource. In this figure, the user has declared the Blindness profile; therefore, the access mode of the resource required for the user must be textual or auditory. In this case, it is supposed that the access required is textual to be interpreted by using screen reader software. The original educational resource has an access mode visual for one of its components, hence, this component can be replaced by an adaptation that has an access mode textual. Therefore, the adapted educational resource only has the access mode textual. The attributes accessMode and accessModeRequired that appear in Figure 1 are part of the AfA standard. This is explained later in section 5.2 Metadata.

\subsection{Integration of design aspects}

The design aspects detailed above are integrated into our OER website as shown in Figure 2.

The selection of a disability profile by the user enables the personalization of the entire system by automatically configuring the accessibility features required for this user. The adaptive interface responds to this profile by enhancing visualization and behavior, considering both accessibility and usability standards. The holistic approach is completed with the IA through metadata, which enables the selection of resources that match the users' needs for access mode, visualization, control, complexity, and language. The aim of this OER environment is to provide an enhancing accessible UX to users.

\section{The OERfAll Website}

OERfAll is the website developed based on this design proposal. For its implementation an educational resource was considering as a learning object that can have one or more components or digital objects of tiny granularity [62]. These resources can be tagged with metadata and stored in the repository for sharing and reusing [63].

\subsection{User personalization}

Some findings of previous works support the use of customization of the web environment to improve accessibility and usability [16-18]. OERfAll allows an automatic personalization of the interface and the content, which conforms to the accessibility requirements of a user through the self-selection of choices of disability and preferred language. Furthermore, the advanced options enable users to configure the "look and feel" of the interface, i.e. their preferences for interface visualization and behavior. Figure 3 presents a screenshot of the homepage of OERfAll with the language selection toolbar on the top right, the disability profile toolbar, and the advanced options button on the bottom. In this screenshot, English language and the user profile for blindness have been selected, so these options are 
Blindness profile

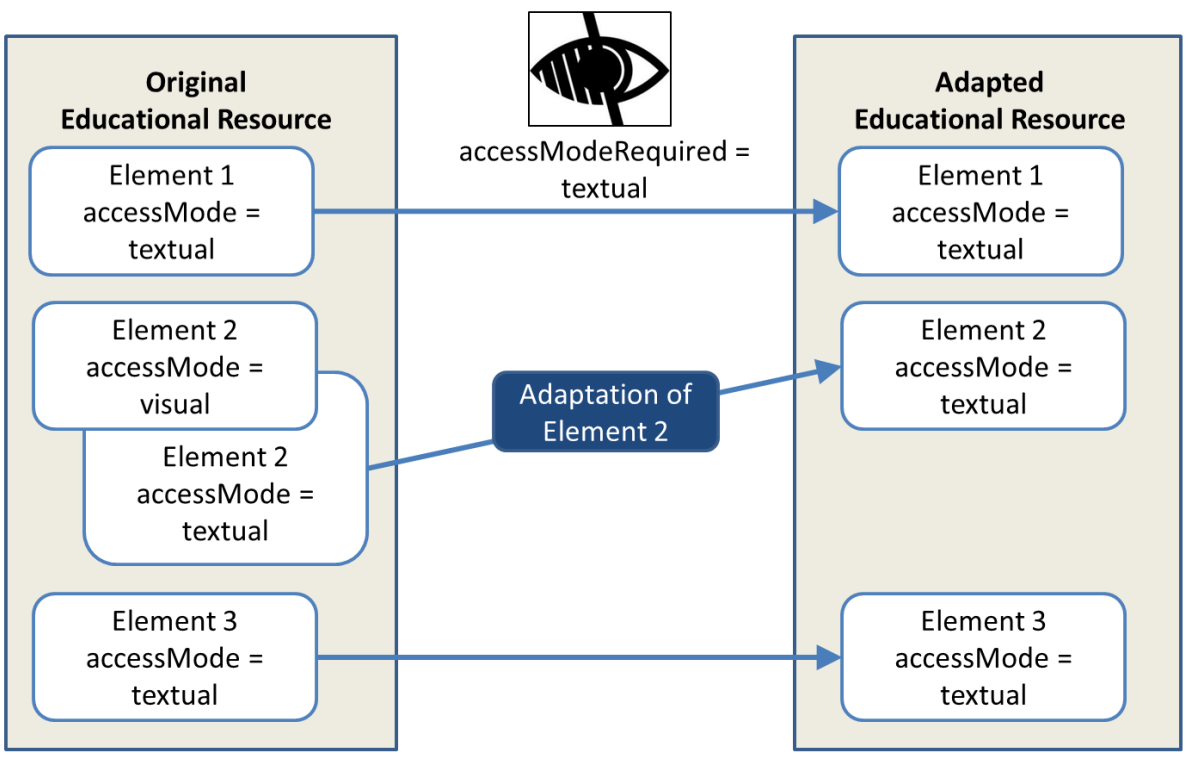

Figure 1: Adaptations for matching user disability profile

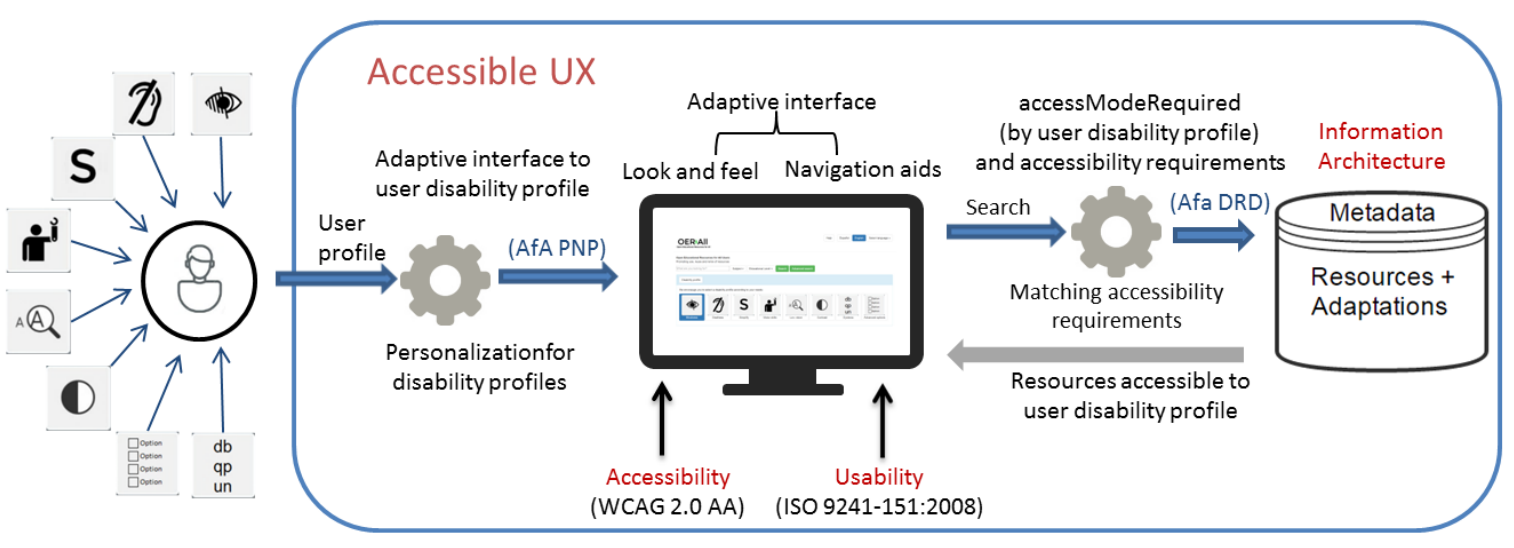

Figure 2: Design aspects of the OER website

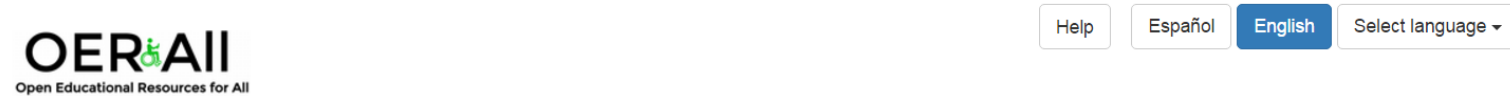

Open Educational Resources for All Users

Promoting use, reuse and remix of resources

\begin{tabular}{ll|l}
\hline Search terms & Subject & Educational Level
\end{tabular}

Search Advanced search

\section{Disability profile}

We encorauge you to select a disability profile according to your needs:

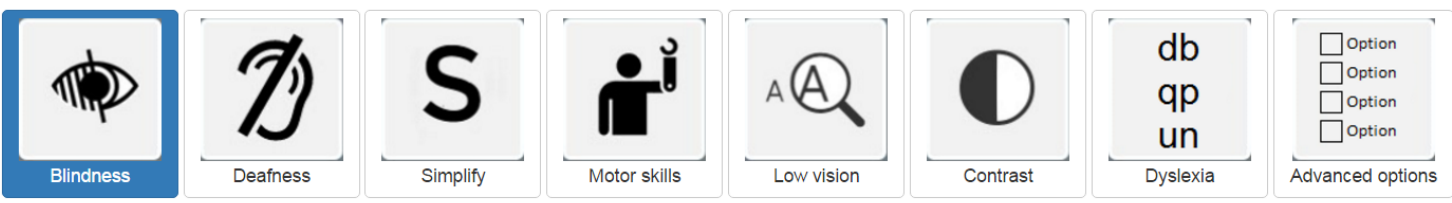

Figure 3: OERfAll homepage 
highlighted. Each element that supports user personalization is explained next.

\subsubsection{Disability profile toolbar}

A disability profile enables the system to customize the interface as required for the respective disability. Also, it defines the resources suitable for the user, based on the access mode of the resources and other accessibility characteristics. The access mode describes how a user can process or perceive information associated with the sensory perceptual system or cognitive faculty required [57]. Once the profile is applied, the user has the possibility of enhancing their choices for the selection of the adaptations of resources by specifying the type of access mode of the required adaptation.

OERfAll offers a disability profile toolbar that enables users to select a disability profile. This profile is kept on the user's computer, by means of HTTP cookies, for future interaction, so the user will not have to configure their profile in each new visit to the website unless he has decided to change it. OERfAll allows the user to select more than one disability profile at the same time; in this case, the features of the combination of these disability profiles are applied. Furthermore, the use of HTTP cookies is transparent to users and exempts them from the mandatory registration process to obtain the benefits of the system.

The options available in the disability profile toolbar (see bottom of Figure 3) are Blindness, Deafness, Simplify, Motor skills, Low vision, Contrast, and Dyslexia. For each disability, the adaptive response of the interface and the restrictions of access mode of educational resources are explained, as follows:

- Blindness. This disability profile selection has no effect on the visualization of the interface. This profile restricts the selection of educational resources whose access mode is related to eyesight. The resources that match this profile are those resources amenable to being explored by employing screen reader software or that have auditory modality to convey their content; for example, an accessible PDF document (PDF/UA) or a video with audio descriptions.

- Deafness. This disability profile selection has no effect on the visualization of the interface. This profile restricts the selection of educational resources whose access mode is related to the sense of hearing. The resources that match this profile are adaptations to the auditory content; for example, podcasts with transcripts, videos with subtitles or with a sign language version.

- Simplify. This profile is intended for users with learning or cognition issues [32]. This profile simplifies the visualization of the interface, shows the table of contents for easy navigation, increases the text size and line spacing and displays only the main content. The resources need to have amenability to transform the font size and the structure presentation. The resources that match this profile are simplified adaptations of the content, i.e., resources that use the simplest language most appropriate to the content.

- Motor skills. This profile is intended for users with motor skills' impairments that hinder them from using a mouse device. The resources that match this profile must enable control by keyboard since most assistive technologies for people with motor disabilities work through the keyboard or by emulating the functionality of the keyboard [64]. This profile increases font size, enlarges the buttons, menus, text fields and other input fields in forms. Besides this, the resources need to have amenability to transform the font size, the layout, and the structure presentation, in order to enable the enlargement of buttons, menus, text fields and other input fields.

- Low vision. This profile is intended for users with low vision who need improving visualization characteristics to overcome their restrictions. This profile enlarges the text of the interface to $200 \%$, maximizes the contrast of the interface and underlines and highlights links [65]. The resources need to have amenability to transform the font size, the font face, the highlighting of presentation and the background and foreground color.

- Contrast. This profile is suitable for users who experience reduced sensitivity to contrast, e.g., older people due to the effects of aging [27]. In such cases, the readability of the content is essential for accessibility. This profile affects the display of the interface: by changing the display of the interface to black on the background and yellow on the foreground. The resources must have amenability to transform the background and foreground color.

- Dyslexia.This profile is intended for users with a neurological disorder called dyslexia which interferes with the acquisition and processing of language. This profile enables a better comprehension of the content by implementing these characteristics: displays the text left-aligned, uses a monospace font type and changes the line spacing to double [29]. Furthermore, this profile applies adaptations concerning the Contrast disability profile. The resources need to have amenability to transform the font size, the font face and the background and foreground colors. 


\section{OERAII}

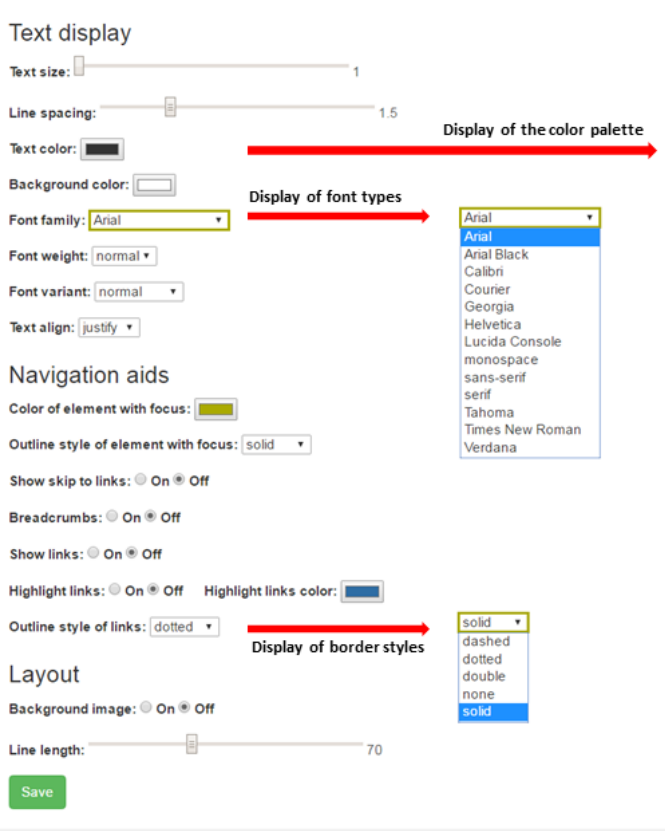

\begin{tabular}{|lll} 
Help Español Englsh Select language - &
\end{tabular}

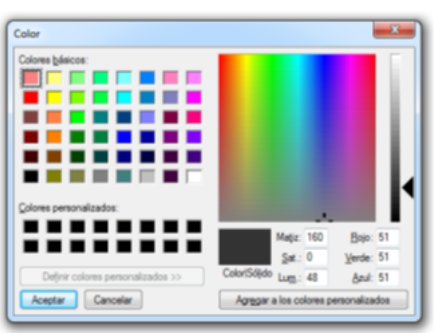

\begin{tabular}{|l|l|l|}
\hline Reset advanced options & Disable profile & Delete profle \\
\hline
\end{tabular}

Figure 4: Advanced options screenshot

\subsubsection{Advanced options}

In addition to the selection of disability profiles, OERfAll offers to users the functionality to adjust their preferences by configuring the following advanced options: text display, navigation aids and layout. This customization is valuable for users without a specific disability who want to define their preferences (e.g. greater text size or different background color) or for users with disabilities who explicitly state their preferences regarding the advanced options. Figure 4 shows the screenshot of advanced options in OERfAll (the screenshot has been modified to show the content of three dropdown lists: Text color, Font family and Outline style of links).

The adjustments provided by each advanced option are explained next:

- Text. Text can be adjusted with regard to these aspects: size, line spacing, color, background color, font (family, weight, variant), and alignment.

- Navigation aids. Navigation aids can be adjusted with regards to the color of elements with focus and the style of their outline; the color to highlight links and the style of their outline. Further, it enables users to show or hide the "skip to links", the breadcrumbs, and the underlining of links.

- Layout. Layout can be adjusted to show or hide the background image and define the maximum num- ber of characters per line. In addition, the layout is based on a responsive web design, so it can automatically adjust to the width of different devices.

\subsubsection{Language toolbar}

Language can be a barrier that hinders the use of the web [66]. OERfAll offers a language toolbar that enables the user to select a preferred language. The language selected is applied to the interface, and is thus considered as the prevalent language for the search of resources.

\subsection{Metadata}

\subsubsection{AfA specification}

The AfA specification is an open standard developed by the IMS Global Consortium [57]; it includes two components: the AfA Digital Resource Description (DRD), which is used to describe the resources and adaptations, and the AfA Personal Needs and Preferences (PNP), which is used to describe users' profiles and needs. These specifications used in conjunction provide a common language that connects accessibility features of resources with users' requirements. 
Table 2: AfA DRD Attributes name and values for descriptors used in OERfAll

\begin{tabular}{|c|c|}
\hline Attribute and Description & Values \\
\hline $\begin{array}{l}\text { accessMode } \\
\text { This term describes how the information conveyed in an educational resource is perceived by the } \\
\text { user by using a sensory modality. This term is associated with the human sensory-perceptual } \\
\text { system; hence, it can be defined by the declaration of disability (blindness, deafness, motor skills } \\
\text { impairments), or a cognitive faculty (if simplified mode is required). For example, accessMode } \\
=\text { auditory means that the user needs the sense of hearing to interpret the information of the } \\
\text { resource. }\end{array}$ & $\begin{array}{l}\text { auditory } \\
\text { color } \\
\text { textOnImage } \\
\text { textual } \\
\text { visual }\end{array}$ \\
\hline $\begin{array}{l}\text { accessModeAdapted } \\
\text { This term describes the accessMode of a resource that is being adapted. For example, if a } \\
\text { resource originally has an accessMode = visual, then for the adaptation of this resource the } \\
\text { accessModeAdapted = visual. }\end{array}$ & $\begin{array}{l}\text { auditory } \\
\text { color } \\
\text { textOnImage } \\
\text { textual } \\
\text { visual }\end{array}$ \\
\hline $\begin{array}{l}\text { adaptationType } \\
\text { This term describes the nature of the adaptation and consequently it defines the accessMode of } \\
\text { the adaptation. For example, if the accessModeAdapted = auditory, and the adaptation Type= } \\
\text { signLanguage, it means that the adaptation is a video with sign language, then the adaptation } \\
\text { will have an accessMode=visual. If the accessModeAdapted = visual and the adaptationType } \\
=\text { longDescription, then the adaptation will be a longer text and their accessMode = textual. }\end{array}$ & $\begin{array}{l}\text { alternativeText } \\
\text { audioDescription } \\
\text { captions } \\
\text { highContrast } \\
\text { longDescription } \\
\text { signLanguage } \\
\text { transcript }\end{array}$ \\
\hline $\begin{array}{l}\text { displayTransformability } \\
\text { This term describes the amenability of a resource to be transformed using styling modification } \\
\text { mechanisms such as Cascading Style Sheets. The display transformability of a resource can } \\
\text { imply modifications in color, size and layout. }\end{array}$ & $\begin{array}{l}\text { fontColour } \\
\text { fontSize } \\
\text { fontFace } \\
\text { backgroundColor } \\
\text { foregroundColor } \\
\text { highlightPresentation } \\
\text { structurePresentation }\end{array}$ \\
\hline $\begin{array}{l}\text { educationalComplexityOfAdaptation } \\
\text { This term describes whether the adaptation of a resource has been simplified or enriched } \\
\text { relative to the original resource. }\end{array}$ & $\begin{array}{l}\text { simplified } \\
\text { enriched }\end{array}$ \\
\hline $\begin{array}{l}\text { languageOfAdaptation } \\
\text { This is the language used in the adapted resource. }\end{array}$ & ISO 639-1 [67] \\
\hline
\end{tabular}

The AfA DRD has a number of descriptors that provide information about resources. Each descriptor has a set of allowable values of a controlled vocabulary. OERfAll has implemented the AfA DRD specification by using the most relevant accessibility descriptors for the purpose of the website, as explained below. Table 2 presents a part of the AfA DRD implemented in the OERfAll website.

The AfA PNP provides information about the profile of the learner regarding their accessibility characteristics and preferences for the resources. The AfA PNP descriptors are stored by means of HTTP cookies for future access.

\subsubsection{Learning Object Metadata}

In order to identify and describe the general characteristics of a resource, OERfAll has used a subset of descriptors from the LOM metadata specification [59] that cover the identification purpose. LOM classifies the data elements to describe a learning resource into nine categories: general, lifecycle, meta-metadata, technical, educational, rights, relation, annotation and classification. Each of these categories includes subcategories. The elements of LOM used in OERfAll are presented in Table 3.

\subsection{Rules for searching resource adaptations}

In OERfAll, the educational resources are stored in such a way that each has a separate metadata instance where the attributes that identify and categorize the resources are presented. A resource is considered as a set of components; each can have more than one access mode. For example, a web page that includes a text and a video has the access modes textual and visual, respectively. These components can be adapted, so the video may have a textual adaptation such as a transcript of the dialogue of the video. In consequence, the model stores 
Table 3: Elements of LOM used in OERfAll

\begin{tabular}{|c|c|c|}
\hline Element & Description & Vocabulary \\
\hline General.Identifier & Unique identification of the resource. & (Catalog.entry) if it exists. \\
\hline General.Title & Title of the resource. & $\begin{array}{l}\text { Any combination of allowed characters } \\
\text { meaningful to the intention of the resource. }\end{array}$ \\
\hline General.Description & Detailed description of the resource. & $\begin{array}{l}\text { Any combination of allowed characters } \\
\text { meaningful to the intention of the resource. }\end{array}$ \\
\hline General.Keywords & Applied to subject area. & $\begin{array}{l}\text { Number of terms or descriptive tags that } \\
\text { characterized the content of the resource. }\end{array}$ \\
\hline General.Language & The language used for the resource. & ISO $639-1[67]$ \\
\hline Technical.Format & Format of the resource. & MIME types [68] \\
\hline Classification.Purpose & Subject area. & ISCED [69] \\
\hline Educational.LearningContext & $\begin{array}{l}\text { Grade or academic level associated } \\
\text { with a resource. }\end{array}$ & ISCED [69] \\
\hline
\end{tabular}

some attributes on the metadata specification concerning their particular adaptation.

The main information concerning the accessibility required by a user is defined through the selection of the disability profile. This profile determines the information about the access mode that should have an educational resource to be perceived by the user as well as the requirements referred to amenability that should have the resource to modify the font size, font face, structured presentation, background color, foreground color and the educational complexity.

We have defined the AFA PNP attributes that should have the resources for each disability profile as presented in Table 4. The system verifies if the AfA DRD of original resource meets these attributes, though if matching fails, then the system searches for adapted resources which comply with these requirements to offer them to the user.

Searching for adapted resources occurs if the original resource does not match the personal needs of the user, represented by the election of a disability profile. More than one adaptation suitable for the user's disability profile could occur. The searching of resources can be expressed through the pseudocode shown in Figure 5 .

The access mode associated with a resource is described with the accessMode attribute and the access mode required for a user (based on their needs or preferences) is described with the accessModeRequired attribute, both attributes defined in AfA. For instance, for Blindness profile, if an original resource has an $a c$ cesssMode = visual or accessMode = textOnImage then the adaptation should have an accessMode = textual or accessMode = auditory. Similarly, for Deafness profile, if an original resource has an accessMode = auditory

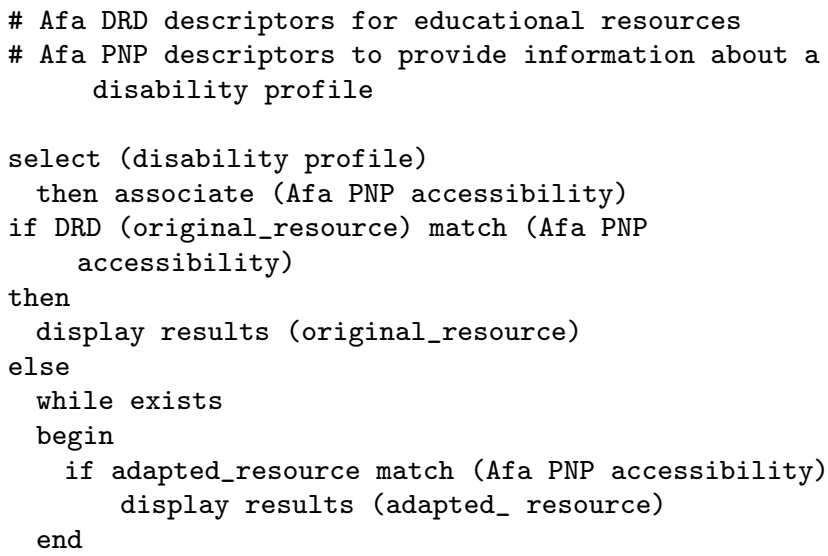

Figure 5: Pseudocode for application of Afa PNP for searching of adapted resources

then the adaptation should have an accessMode $=$ textual or accessMode = visual.

For other disability profiles, adaptations could require adjusting the educational complexity of the resource, the interaction by using the keyboard and the amenability to transform the display of the resource.

Furthermore, the selection of language is available through the language toolbar on the homepage and it is considered part of the profile; hence, it is defined by the AfA PNP attribute languageOfAdaptation.

\subsection{Example of use cases}

By way of example, the functionality of OERfAll is shown in two use cases: for a Blindness profile and for a Simplify profile. 
Table 4: Personalization based on user disability profile matching AfA PNP

\begin{tabular}{|c|c|}
\hline Disability profile & Personal needs \\
\hline Blindness & $\begin{array}{l}\text { accessModeRequired }=\text { textual } \\
\text { accessModeRequired }=\text { auditory }\end{array}$ \\
\hline Deafness & $\begin{aligned} \text { accessModeRequired } & =\text { textual } \\
\text { accessModeRequired } & =\text { visual }\end{aligned}$ \\
\hline Simplify & $\begin{array}{l}\text { educationalComplexityOfAdaptation }=\text { simplified } \\
\text { displayTransformability }=\text { fontSize } \\
\text { displayTransformability }=\text { structuredPresentation }\end{array}$ \\
\hline Motor skills & $\begin{array}{l}\text { inputRequirements }=\text { fullKeyboardControl } \\
\text { displayTransformability }=\text { fontSize } \\
\text { displayTransformability }=\text { layout } \\
\text { displayTransformability }=\text { structuredPresentation }\end{array}$ \\
\hline Low vision & $\begin{array}{l}\text { displayTransformability }=\text { fontSize } \\
\text { displayTransformability }=\text { fontFace } \\
\text { displayTransformability }=\text { highlightPresentation } \\
\text { displayTransformability }=\text { backgroundColor } \\
\text { displayTransformability }=\text { foregroundColor }\end{array}$ \\
\hline Contrast & $\begin{array}{l}\text { displayTransformability }=\text { backgroundColor } \\
\text { displayTransformability }=\text { foregroundColor }\end{array}$ \\
\hline Dyslexia & $\begin{array}{l}\text { displayTransformability }=\text { backgroundColor } \\
\text { displayTransformability }=\text { foregroundColor } \\
\text { displayTransformability }=\text { fontSize } \\
\text { displayTransformability }=\text { fontFace }\end{array}$ \\
\hline
\end{tabular}

\subsubsection{Case 1: A user with Blindness profile}

Initially, the user has activated the Blindness profile. He has searched resources by using the "global warming" query. Figure 6 shows the information of a selected resource from the delivered resources to this user profile in the search results.

This resource has been presented to this profile due to its adaptations. The adapted resource is displayed by default; however, the user can select "View resource" to examine the original resource; for example, if the user is working with a sighted person. Figure 7 shows this resource in its original format containing two components, text and video, and Figure 8 presents an adaptation to the Blindness profile that correspond only to accessModeRequired $=$ textual because a transcript has replaced the video. The first element of the original resource does not need an adaptation.

\subsubsection{Case 2: A user with Simplify profile}

Initially, the user has activated the Simplify profile and has searched resources by using the phrase "human rights" for the query. Figure 9 shows the information of a selected resource. The visualization of the interface has been affected by the user profile; the text size and line spacing have been increased and the breadcrumbs menu is visible below the toolbar.
By default, the adapted resource is displayed; however, the user can select "View resource" to visualize the original resource. Figure 10 shows this resource, an accessible PDF document with its original content, and Figure 11 presents an adaptation that shows the content by using plain language [70] and highlighting the main ideas of the text, in such a way that can be clearly understood by all people, even by people with a cognitive disability or with a low educational level.

\section{Evaluation of OERfAll}

To test the validity of the approach to accessibility implemented in the OERfAll website we have carried out two kinds of evaluation. On the one hand, an accessibility evaluation was conducted by using web accessibility evaluation tools. On the other hand, a set of user tests were carried out with users who have different disabilities. To make a comparison, these evaluations have been applied on OERfAll and OER Commons, a well-known OER website.

\subsection{Accessibility evaluation by using automatic tools}

OER Commons and OERfAll were examined by using four accessibility evaluation tools: Wave, eXaminator, TAW, and Achecker. In all of these tools the 


\section{OERAII}

Earth System: Ice and Global Warming

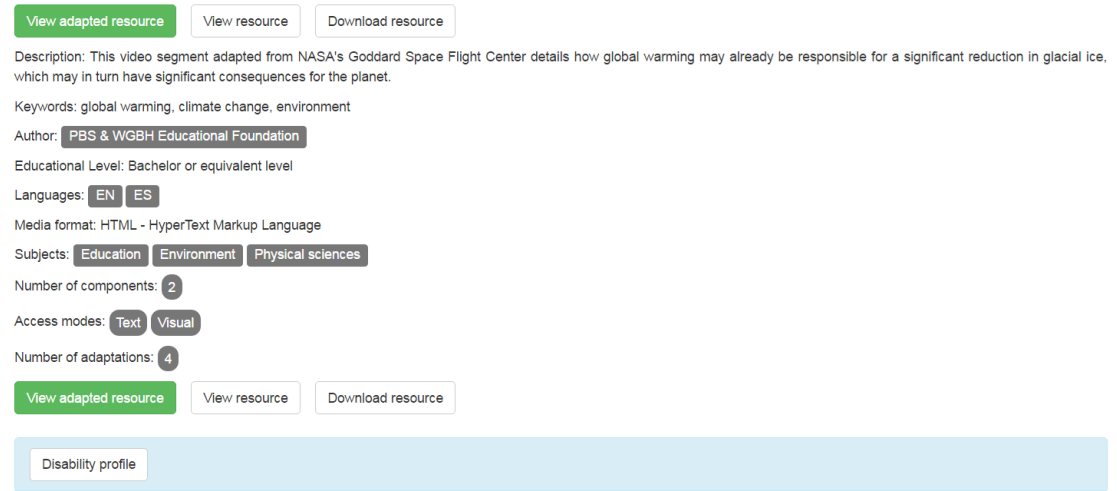

Figure 6: Information of the resource that contains adaptations for Case 1

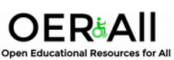

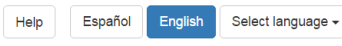

Earth System: Ice and Global Warming

Scientific evidence strongly suggests that different regions on Earth do not respond equally to increased temperatures. Ice-covered regions appear to be particularly sensitive to even
small changes in global temperature. This video segment adapted from NASA's Goddard Space Flight Center detalls how global warming may already be responsille for a significant reduction in glacial ice, which may in turn have significant consequences for the planet.
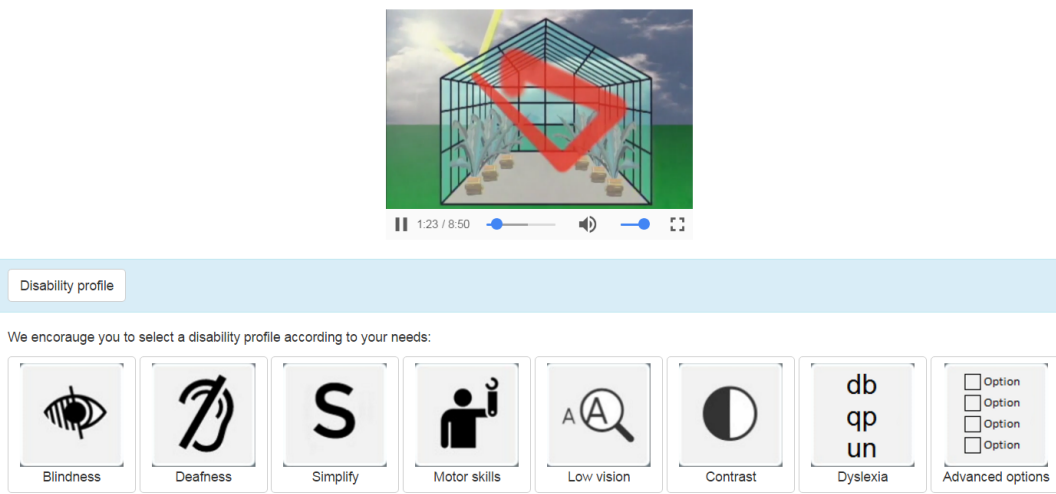

Figure 7: The resource in its original format for Case 1

OERAAll

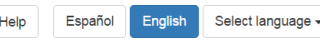

Earth System: Ice and Global Warming

Scientific evidence strongly suggests that different regions on Earth do not respond equally to increased temperatures. Ice-covered regions appear to be particularly sensitive to even
small changes in global temperature. This video segment adapted from NASA's Goddard Space Flight Center detalls how global warming may already be responsilibe for a significant TRANSCRIPT

Glaciers exist on all continents except Australia and at vitually all latitudes from the tropics to the poles. Mountain glaciers, such as those that exist at higher elevations in the midattitudes and tropics, are particularly sensitive indicators of climate change. The volume of ice in a glacier and correspondingly its surface area, thickness, and length is determined by the balanne between inputs (accumulation of snow and ice) and outputs (melting and calving). As climate changes, the balance between inputs and outputs may change, resulting in a
change in thickness and the advance or retreat of the glacier. Temperature, precipitation, humidity, wind speed, and ther factors such as slope and the reflectivity of the glacier surface all affect the balance between inputs and outputs. Most glaciers in the world, however, are more sensitive to temperature than to other climatic factors.

Disability profle

Figure 8: The resource adapted to Blindness profile for Case 1 
OER All

\section{Home / Results / Resource}

\section{Declaration of Human Rights}

\section{View adapted resource $\quad$ View resource}

Description: The Universal Declaration of Human Rights (UDHR) is a compromise adopted by the United Nations to ensure the enjoyment of the fundamental rights for people.

Keywords: human rights, declaration of human rights

Figure 9: Information of the resource that contains adaptations for Case 2

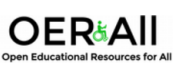

Help Españo
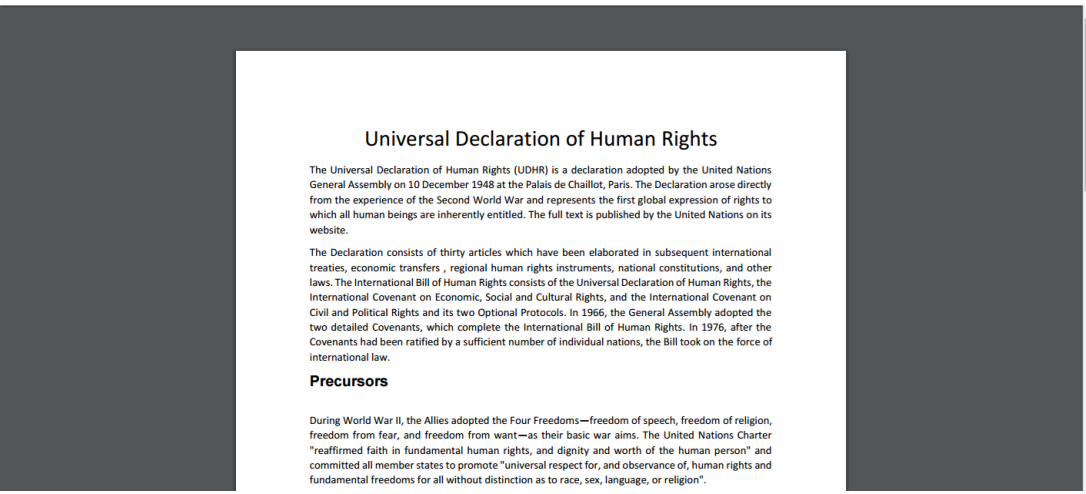

Figure 10: Resource with its original content for Case 2
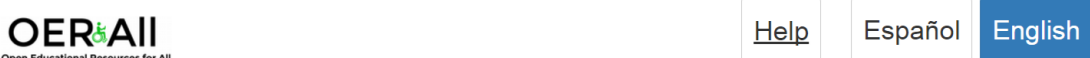

Select language .

Home / Results / Resource

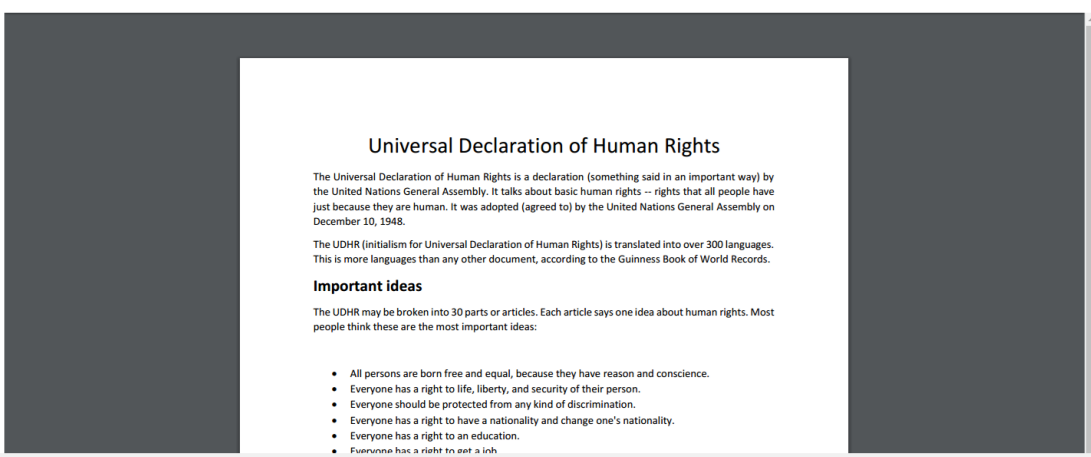

Figure 11: Resource adapted for Simplify profile for Case 2 


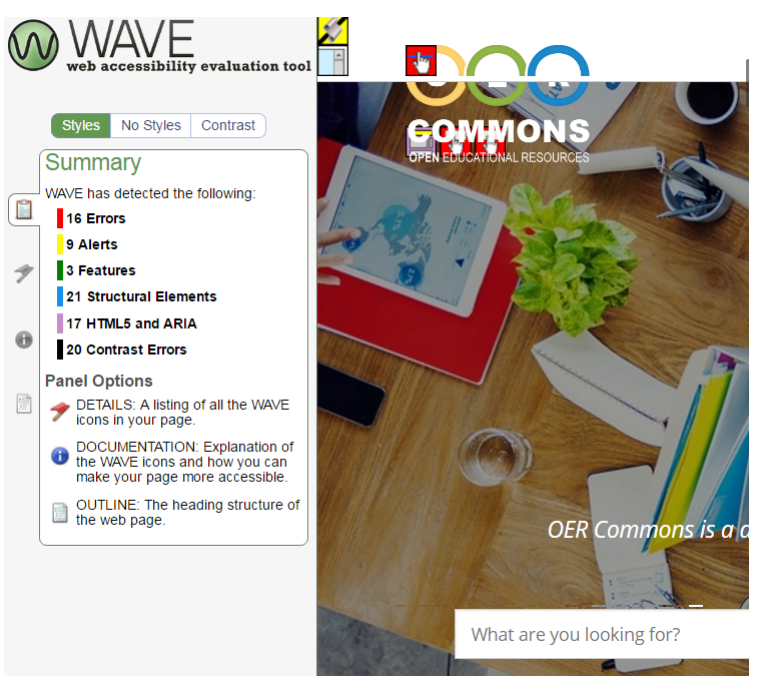

(a) OERCommons

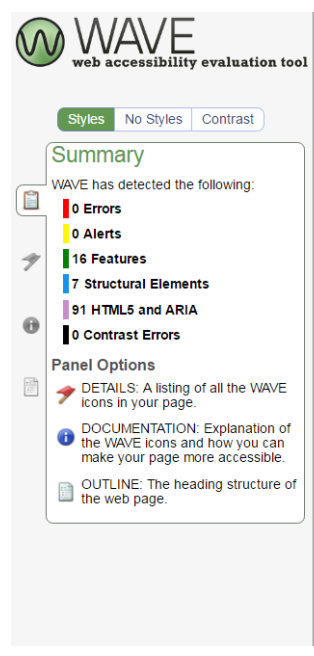

(b) OERfAll
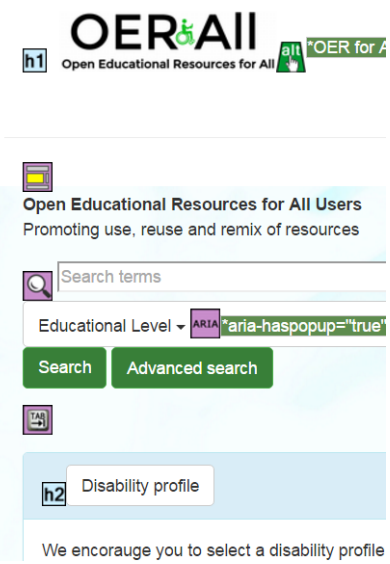

We encorauge you to select a disability profile

Figure 12: Accessibility evaluation by using WAVE

evaluation for OERfAll did not report accessibility issues, while some accessibility problems were detected on OER Commons. Due to the lack of space, only the evaluation with Wave is reported here. Figure 12 shows a partial screenshot of the accessibility evaluation by using Wave on the homepage of both websites. It is observed that (a) OER Commons presents accessibility errors, however (b) OERfAll does not present any accessibility error.

It is of primary importance that the homepage of the website complies with accessibility principles of WCAG 2.0 at level AA in order to enable users their first interaction with the website. In the case of OERfAll, the accessibility evaluation results of the homepage confirm that users do not face accessibility problems that hinder them from selecting their profile from the disability profiles' toolbar.

\subsection{UX evaluation}

UX testing has been conducted to consider the user's perspective regarding the validity of our approach to accessibility. In the same way, UX testing has been applied to two websites: OERfAll and OER Commons.

\subsubsection{Participants}

The number of participants for usability and UX testing has been extensively discussed in research works. The assumption that five users are sufficient for usability testing comes from Nielsen [71,72] and Virzi [73]. Nevertheless, another work has argued that increasing the number from five to ten users obtains an improvement in confidence in the results [74], while others have proposed the rule of ten users plus two for each segment of the audience [75]. Furthermore, the idea that the number of users for testing should increase with the number of heterogeneous groups is stated in [76].

In summary, there is no consensus among researchers regarding the correct number of users for testing; hence, for this first evaluation, we have considered as meaningful the evaluation conducted with twenty users with different disabilities. Users have self-identified with the profiles: Blindness, Motor skills, Low vision, Contrast, Simplify, and Dyslexia. To participate in this testing, users needed to have experience of at least five years in web interaction and proficiency in the English language. Users were recruited as volunteers by the Council of Disabilities of Ecuador and the Association of Retirement Teachers of Chicago, USA.

\subsubsection{Procedure}

All test sessions were conducted in the workplace of each user and they used the assistive technology of their preference (one which they usually use). The users' testing included two common basic tasks for OER websites as described below:

1. Search for resources using the phrase "global warming" for the query. Inform the number of resources reported in search results.

2. Search for resources on the "Education" subject. Retrieve the first resource from the search results' list and write the first phrase of the resource content. 
The procedure for the UX test included the following steps:

- Perform the tasks. Users carry out both of the tasks on each website. For each task, users need to access the website (the website is closed when the first task is concluded). The time for completion or abandonment is counted from access to the website until the task is completed or abandoned.

- Complete the UX questionnaire. After performing the tasks, users evaluate their UX through a questionnaire that examines their perceptions on three UX quality aspects, namely perspicuity, efficiency and dependability [45], in addition to the aspect of accessibility. For each aspect, at least two questions were included. Users graded the task accomplishment for each item on the aforementioned aspects, using a scale of seven values ranging from -3 (very bad) to 3 (very good), with 0 representing a neutral opinion.

- Comment upon their experience. All users were requested to express their views about the positive and negative aspects of the accessibility of both websites, and their opinion about the helpfulness of the disability profiles and the adaptation of educational resources. They also provided feedback on their experience on each website.

\subsubsection{Questionnaire for evaluation}

The questions included for each aspect presented a descriptive phrase or adjective to add meaning to the opposite values (from less to more). They addressed the following:

- Perspicuity. Is it easy to learn how to use the website?

- Q1. How easy was it for you to learn how to use the website? (difficult to learn / easy to learn).

- Q2. How understandable do you find the structure of the website? (not understandable / understandable).

- Efficiency. Can the user resolve the tasks on the website without unnecessary effort?

- Q3. How efficient do you find the navigation in relation to your purpose on the website? (inefficient / efficient).

- Q4. How easy do you find the process on the website for the achievement of the task? (complicated / easy).

- Dependability. Does the user feel in control of interaction on the website?

- Q5. How predictable do you find the control of interaction on the website? (unpredictable / predictable).
- Q6. How do you feel about the control of interaction on the website? (not secure / secure).

- Accessibility. How adequate is the website for a user's particular accessibility requirements?

- Q7. How supportive do you feel the website is of your accessibility requirements? (obstructive / supportive).

- Q8. What is your experience of this website with regard to your accessibility requirements? (does not meet expectations / meets expectations).

- Q9. How helpful is the adaptability for your interaction requirements? (useless / very helpful).

\subsubsection{UX Results and discussion}

Table 5 shows information about the users and the time that they invested for the achievement of the proposed tasks during the UX testing. Although all users had experience of web interaction, none of them had used an OER website before this test however, in spite of that, all users completed the tasks.

Regarding the assistive technology, only blind users used screen reader software for navigation on the websites. The following information details the screen reader software and the years of experience of each blind user in using this technology: blind user 1 (NVDA, 7 years); blind user 2 (JAWS, 15 years); blind user 3 (JAWS, 20 years); blind user 4 (JAWS, 5 years) and blind user 5 (JAWS, 20 years).

Not all users who were volunteers for testing could meet the requirements; this is the reason for the reduced number of users in some profiles.

The average time for task completion in both websites, considering the times registered by the users, was very close. It is worth highlighting that blind users completed the tasks in the shortest time (by using JAWS as the screen reader software). This is likely explained due to the fact that they did not invest time in examining the visual disposition of elements as the others users did. The average time required by a user with a learning / cognitive disability exceeded the average time of users with other disabilities.

Regarding the results of the UX questionnaire, Figure 13 shows a bar graph with a comparison of the average score of the questions for each aspect of the UX on both websites. The results show that the difference in the evaluation of perspicuity and efficiency are minimally in favor of OER Commons (3.68\%, and $8.33 \%$ respectively); dependability is best assessed on OER Commons (28.04\% more); nevertheless, the accessibility evaluation on OERfAll exceeds, in a considerable proportion, OER Commons (169.01\%). These results 
Table 5: Users information and time for task achievement

\begin{tabular}{|c|c|c|c|c|c|c|c|c|c|}
\hline \multirow[t]{2}{*}{$\#$} & \multirow[t]{2}{*}{ User disability } & \multirow[t]{2}{*}{ Age } & \multirow[t]{2}{*}{ Gender } & \multirow[t]{2}{*}{ Studies } & \multirow{2}{*}{$\begin{array}{c}\text { Web } \\
\text { experience } \\
\text { (years) }\end{array}$} & \multicolumn{2}{|c|}{$\begin{array}{l}\text { OER Commons } \\
\text { (minutes) }\end{array}$} & \multicolumn{2}{|c|}{$\begin{array}{c}\text { OERfAll } \\
\text { (minutes) }\end{array}$} \\
\hline & & & & & & Task1 & Task2 & Task1 & Task2 \\
\hline 1 & $\begin{array}{l}\text { Motor skills } 1 \\
\text { (muscular dystrophy) }\end{array}$ & 65 & Male & Bachelor & 25 & 6 & 7 & 4 & 4 \\
\hline 2 & $\begin{array}{l}\text { Motor skills } 2 \\
\text { (arthritis) }\end{array}$ & 49 & Female & Bachelor & 15 & 3 & 3 & 2 & 3 \\
\hline 3 & $\begin{array}{l}\text { Motor skills } 3 \\
\text { (arthritis) }\end{array}$ & 57 & Female & Secondary & 12 & 5 & 7 & 4 & 6 \\
\hline 4 & $\begin{array}{l}\text { Motor skills } 4 \\
\text { (dyspraxia) }\end{array}$ & 18 & Male & Secondary & 8 & 6 & 5 & 3 & 6 \\
\hline 5 & $\begin{array}{l}\text { Low vision } 1 \\
\text { (presbyopia) }\end{array}$ & 68 & Male & Postgraduate & 20 & 3 & 4 & 3 & 4 \\
\hline 6 & $\begin{array}{l}\text { Low vision } 2 \\
\text { (cataracts) }\end{array}$ & 66 & Male & Bachelor & 20 & 4 & 5 & 3 & 4 \\
\hline 7 & $\begin{array}{l}\text { Low vision } 3 \\
\text { (cataracts) }\end{array}$ & 59 & Female & Bachelor & 25 & 5 & 4 & 4 & 6 \\
\hline 8 & $\begin{array}{l}\text { Low vision } 4 \\
\text { (diabetic retinopathy) }\end{array}$ & 53 & Male & Bachelor & 12 & 4 & 3 & 4 & 4 \\
\hline 9 & $\begin{array}{l}\text { Contrast } \\
\text { (presbyopia) }\end{array}$ & 60 & Male & Bachelor & 15 & 6 & 6 & 5 & 6 \\
\hline 10 & $\begin{array}{l}\text { Simplify } \\
\text { (down syndrome) }\end{array}$ & 26 & Female & Secondary & 10 & 8 & 10 & 10 & 15 \\
\hline 11 & $\begin{array}{l}\text { Simplify } \\
\text { (old person) }\end{array}$ & 72 & Male & Postgraduate & 20 & 5 & 4 & 3 & 5 \\
\hline 12 & Dyslexia 1 & 16 & Female & Secondary & 8 & 1 & 2 & 1 & 2 \\
\hline 13 & Dyslexia 2 & 21 & Male & Secondary & 12 & 2 & 2 & 1 & 2 \\
\hline 14 & Blindness 1 & 41 & Male & Bachelor & 10 & 5 & 8 & 5 & 5 \\
\hline 15 & Blindness 2 & 47 & Male & Postgraduate & 25 & 1 & 1 & 2 & 2 \\
\hline 16 & Blindness 3 & 37 & Male & Bachelor & 22 & 1 & 1 & 1 & 3 \\
\hline 17 & Blindness 4 & 32 & Male & Secondary & 8 & 7 & 10 & 4 & 6 \\
\hline 18 & Blindness 5 & 30 & Male & Bachelor & 30 & 2 & 3 & 3 & 5 \\
\hline 19 & Deafness 1 & 25 & Female & Secondary & 10 & 3 & 2 & 1 & 2 \\
\hline \multirow[t]{2}{*}{20} & Deafness 2 & 34 & Female & Secondary & 8 & 2 & 3 & 2 & 4 \\
\hline & & & & Average cor & tion time: & 4.0 & 4.5 & 3.3 & 4.7 \\
\hline
\end{tabular}

are considered auspicious for the design proposal presented in this paper that aims to bridge the accessibility gap in OER environments.

Some positive aspects of OERfAll highlighted by users include their satisfaction regarding the accessibility issues that have been explicitly addressed:

- Users with Dyslexia and Down syndrome agreed upon the helpfulness of the profile Simplify for their accessibility requirements. They valued the navigation aids (in this case, the visualization of the table of contents).

- Four older people who selected the Low vision profile expressed their satisfaction about the enlarged fonts and highlighted links.

- Two older people highlighted the selection of the language (for the interface and resources) as an equity feature for people who are not native English speakers.

- Thirteen users recognized as positive that the profile needed to be defined only for the initial access because it is retained for the next (70\%). These users belong to following profiles: Motor skills (one user); Low vision (four users); Simplify (two users); Dyslexia (one user); Blindness (three users); Deafness (one user) and Contrast (one user). Other users did not give an opinion on this topic.

- Twelve users recognized the helpfulness of the concept of resources' adaptation to get suitable resources for their profiles $(60 \%)$. These users belong to the following profiles: Motor skills (two users); Low vision (two users); Simplify (two users); Dyslexia (two users); Blindness (three users) and Deafness (one user). Meanwhile, four users considered that this was a confused concept; they belong to the following profiles: Motor skills (one user); Blindness (two users), Deafness (one user). Four users did not give an opinion on this topic.

Moreover, two users with the Blindness profile expressed their preference for the universal design with respect to the adaptive design based on the selection 


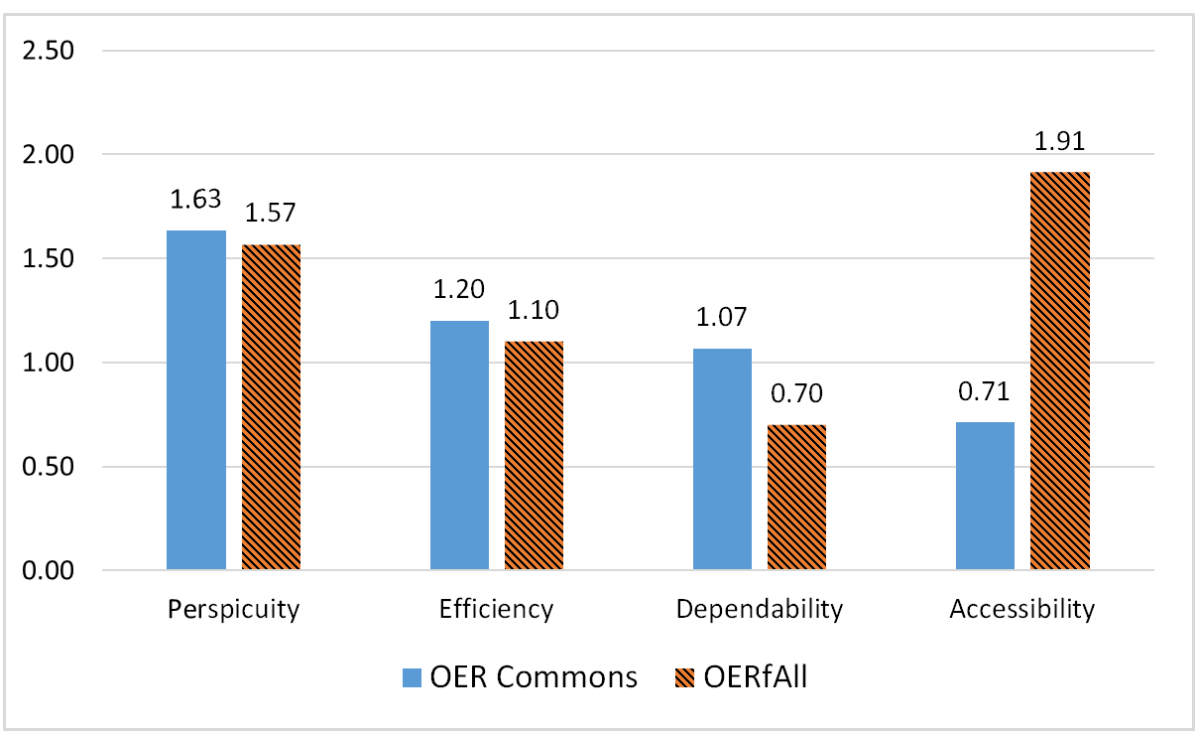

Figure 13: Comparison of the scores of UX aspects on OER Commons and OERfAll

of a disability profile. They disagreed with the need to declare their disability by means of the Disability profile toolbar. In particular, one of them said that the selection of educational resources that would match his disability could be useful when navigating alone, but not with the help of a supporting person. By the way, although the adapted resources matching users' profile needs are presented as the default way, OERfAll enables users to view all resources, not only those adapted or suitable to their profile. This is shown in Figure 6 and Figure 9 where the page of resource information includes the options "View adapted resource" and "View resource".

Some recommendations for improvement were related to the semantics. For example, a blind user suggested replacing the heading "Filter panel" (to filter search results) with the heading "Modify your search results", and another blind user suggested replacing "Skip to results" with the heading "Go to results". Two elderly users recommended the inclusion of the disability profile "Old age".

Finally, as the evaluations were graded on a scale from -3 (very bad) to 3 (very good), it is notable that accessibility has the highest score as the most influential aspect on the UX. These results enable us to argue that the efforts for accessibility issues focused on the UX perspective are appreciated by users.

\section{Conclusion}

The integrative approach of accessibility proposed aims to create an equitable and inclusive OER environment focused primarily on the explicit recognition of accessibility needs of people with disabilities. This design enables users' personalization through their disability profiles. The adaptive response of the system enhances accessibility characteristics in accordance with the profile; therefore, it improves UX. Personalization exempts users from investing time and effort in the configuration of accessibility options that they require. Furthermore, personalization also includes the searching of educational resources that match perceptions modality and the particular needs of users for acquiring information.

In addition, this proposed design aims to support an accessible UX that goes beyond compliance with the ISO/IEC 40500 WCAG 2.0 and incorporates usability aspects and IA, intended to support the delivering of educational resources suitable for the user, based on the availability of adaptations of educational resources cataloged with their own metadata.

The evaluation results of the OERfAll website, developed as a proof of concept, confirm the assumptions of our work. This website ensures the access, interaction, search and retrieval of educational resources, according to a profile based on a user's disability and preferences. On the one hand, we have ensured that accessibility on the homepage of the OERfAll website enables users to firstly, interact and consequently, select their disability profile to guide personalization. On the other hand, the high score obtained by OERfAll in accessibility aspects of the UX testing (1.91) proves that users value the proposed approach to enhance accessibility. This value significantly exceeds the score obtained for the website for comparison, OER Commons (0.71). Another positive aspect is the approval from $60 \%$ of users 
regarding the search of educational resources adapted to their needs of perception and the accessibility requirements.

Moreover, in comparison with the current state of OER websites, OERfAll achieves a clear advantage regarding accessibility personalization, which enhances UX of users with regards to their disabilities.

Finally, the approach presented in this work, intended to enhance UX focusing in users with disabilities, can be applied to other web environments. For instance, by adding a disability profile to an online newspaper it could configure the interface for users with cognitive impairment and could serve transcriptions instead of videos to blind users.

As future work, we propose to study the impact of this approach regarding the privacy and acceptance of users. Besides that, we plan to extend the UX testing on OERfAll, to improve the fine tuning of the environment by including not only each disability but also the assistive technology and the user's expertise level on the web.

\section{References}

1. United Nations (2015), Sustainable Development Goals 2030 Agenda - Goal 4: Quality Education. http://goo. gl/4xZ7ip.

2. UNESCO (2015), Education for All. http://goo.gl/ L8phCw.

3. UNESCO (2002), Forum on the Impact of Open Courseware for Higher Education in Developing Countries: Final report. http://goo.gl/26HSvR.

4. Atkins, D. E., Brown, J. S., and Hammond, A. L. (2007) $A$ review of the Open Educational Resources (OER) Movement: Achievements, Challenges, and New Opportunities. The William and Flora Hewlett Foundation.

5. Cape Town Meeting (2007), Cape Town Open Education Declaration: Unlocking the promise of open educational resources.

6. Wiley, D., Caswell, T., Henson, S., and Jensen, M. (2008) Open Educational Resources: Enabling universal education. The International Review of Research in Open and Distributed Learning, 9, 1-18.

7. UNESCO (2012), World Open Educational Resources (OER) Congress. http://goo.gl/3lhjJG.

8. Navarrete, R. and Luján-Mora, S. (2013) Accessibility considerations in Learning Objects and Open Educational Resources. Proceedings of the 6th International Conference of Education, Research and Innovation (ICERI), Sevilla, Spain, pp. 521-530.

9. Navarrete, R. and Luján-Mora, S. (2015) Evaluating accessibility of Open Educational Resource Website with an Heuristic Method. 9th International Technology, Education and Development Conference (ITHED), Madrid, Spain, pp. 6402-6412.

10. Navarrete, R. and Lujan-Mora, S. (2015) Evaluating findability of Open educational Resources from the perspective of users with disabilities: A preliminary approach. Second International Conference on eDemocracy 83 eGovernment (ICEDEG), Quito, Ecuador, pp. 112119, IEEE.
11. W3C (2012), Diversity of web users. http://www.w3.org/ WAI/intro/people-use-web/diversity.

12. World Health Organization (2014), Visual impairments and blindness. http://www.who.int/mediacentre/ factsheets/fs282/en/.

13. World Health Organization (2015), Deafness and hearing loss. http://www . who. int/mediacentre/factsheets/ fs $300 / e n /$.

14. United Nations (2015), World Population Ageing. http: //goo.gl/P73vyd.

15. United Nations (2013), High-level meeting of the General Assembly on disability and development. http:// www.un.org/en/ga/68/meetings/disability/.

16. Treviranus, J., Mitchell, J., Clark, C., and Roberts, V. (2014) An Introduction to the FLOE project. International Conference on Universal Access in HumanComputer Interaction, pp. 454-465, Springer.

17. Kelly, B., Nevile, L., Sloan, D., Fanou, S., Ellison, R., and Herrod, L. (2009) From Web Accessibility to Web Adaptability. Disability and Rehabilitation: Assistive Technology, 4, 212-226.

18. Cooper, M., Sloan, D., Kelly, B., and Lewthwaite, S. (2012) A Challenge to Web Accessibility Metrics and Guidelines: Putting People and Processes First. Proceedings of the international cross-disciplinary conference on Web accessibility, p. 20, ACM.

19. Gkatzidou, V. and Pearson, E. (2010) Exploring the development of adaptable learning objects. A practical approach. (ICALT), 2010 10th IEEE International Conference on Advanced Learning Technologies, pp. 307-309, IEEE.

20. Gkatzidou, V. and Pearson, E. (2011) Exploring a Standards-based Approach to the Design and Delivery of Adaptable Learning Content: A Case Study. (ICALT), 2011 11th IEEE International Conference on Advanced Learning Technologies, pp. 267-271, IEEE.

21. Mirri, S., Pantieri, D., Roccetti, M., and Salomoni, P. (2006) A Multimedia Broker for Accessible Learning Objects Transcoding. 2006 2nd International Conference on Information \& Communication Technologies, vol. 1, pp. 641-646, IEEE.

22. Kourbetis, V. and Boukouras, K. (2014) Accessible Open Educational Resources for Students with Disabilities in Greece: They are Open to the Deaf. International Conference on Universal Access in Human-Computer Interaction, pp. 349-357, Springer.

23. Borgia, F., Bianchini, C. S., and De Marsico, M. (2014) Towards Improving the e-learning Experience for Deaf Students: e-LUX. International Conference on Universal Access in Human-Computer Interaction, pp. 221-232, Springer.

24. Power, C., Freire, A., Petrie, H., and Swallow, D. (2012) Guidelines are Only Half of the Story: Accessibility Problems Encountered by Blind Users on the Web. Proceedings of the SIGCHI conference on human factors in computing systems, pp. 433-442, ACM.

25. Ferati, M., Vogel, B., Kurti, A., Raufi, B., and Astals, D. S. (2012) Web Accessibility for Visually Impaired People: Requirements and Design Issues. International Workshop on Usability-and Accessibility-Focused Requirements Engineering, pp. 79-96, Springer.

26. Radovan, M. and Perdih, M. (2016) Developing Guidelines for Evaluating the Adaptation of Accessible WebBased Learning Materials. The International Review of Research in Open and Distributed Learning, 17.

27. Rello, L. and Baeza-Yates, R. (2012) Optimal colors to improve readability for people with dyslexia. Text Customization for Readability Online Symposium. 
28. Zorzi, M., Barbiero, C., Facoetti, A., Lonciari, I., Carrozzi, M., Montico, M., Bravar, L., George, F., PechGeorgel, C., and Ziegler, J. C. (2012) Extra-large letter spacing improves reading in dyslexia. Proceedings of the National Academy of Sciences, 109, 11455-11459.

29. Rello, L. and Baeza-Yates, R. (2013) Good fonts for dyslexia. Proceedings of the 15th International ACM SIGACCESS Conference on Computers and Accessibility, p. 14, ACM.

30. de Santana, V. F., de Oliveira, R., Almeida, L. D. A., and Ito, M. (2013) Firefixia: An Accessibility Web Browser Customization Toolbar for People with Dyslexia. Proceedings of the 10th International Cross-Disciplinary Conference on Web Accessibility, pp. 16:1-16:4, ACM.

31. Spalteholz, L., Li, K. F., Livingston, N., and Hamidi, F. (2008) Keysurf: A Character Controlled Browser for People with Physical Disabilities. Proceedings of the 17th international conference on World Wide Web, pp. 31-40, ACM.

32. Small, J., Schallau, P., Brown, K., and Appleyard, R. (2005) Web Accessibility for People with Cognitive Disabilities. CHI '05 Extended Abstracts on Human Factors in Computing Systems, New York, NY, USA, pp. 17931796, CHI EA '05, ACM.

33. de Lara, S. M. A., Fortes, R. P. d. M., Russo, C. M., and Freire, A. P. (2016) A study on the acceptance of website interaction aids by older adults". Universal Access in the Information Society, 15, 445-460.

34. Boni, M., Cenni, S., Mirri, S., Muratori, L. A., and Salomoni, P. (2006) Automatically producing IMS AccessForAll Metadata. Proceedings of the 2006 international cross-disciplinary workshop on Web accessibility (W4A): Building the mobile web: rediscovering accessibility?, pp. 92-97, ACM.

35. Cheetham, A., Ayotte, D., Hung, J., Vass, J., Clark, C., Mitchell, J., and Treviranus, J. (2014) Accessible metadata generation. International Conference on Universal Access in Human-Computer Interaction, pp. 101-110, Springer.

36. Little, S., Mikroyannidis, A., Okada, A., and Scott, P. (2011) Formal Metadata and Shared Experiences for Discovering Tools to Adapt Open Educational Resources. Signal-Image Technology and Internet-Based Systems (SITIS), 2011 Seventh International Conference on, pp. 147-153, IEEE.

37. Navarrete, R. and Luján-Mora, S. (2014) Metadata in Open Educational Resources Websites: A review from the perspective of disabled users' requirements. International Conference on Education and New Learning Technologies, Barcelona, Spain, pp. 111-120.

38. Freire, A. P., de Bettio, R. W., Frade, E. G., Ferrari, F. B., Monserrat Neto, J., and Libardi, H. (2013) Accessibility of web and multimedia content: techniques and examples from the educational context. Proceedings of the 19th Brazilian symposium on Multimedia and the web, pp. 7-8, ACM.

39. Navarrete, R., Luján-Mora, S., and Peñafiel, M. (2016) Enhancing User Experience of Users with Disabilities: Application to Open Educational Resources Websites. The Ninth International Conference on Advances in Computer-Human Interactions (ACHI), Venice, Italy, pp. 11-16.

40. Navarrete, R. and Luján-Mora, S. (2016) Improving OER Websites for Learners with Disabilities. Proceedings of the 13th Web for All Conference, p. 16, ACM.
41. Horton, S. and Quesenbery, W. (2014) A web for everyone: Designing accessible User Experience. Rosenfeld Media.

42. The Paciello Group (2013), Accessible User Experience and W3C-WAI. https://goo.gl/xK96Do/.

43. Law, E. L.-C., Roto, V., Hassenzahl, M., Vermeeren, A. P., and Kort, J. (2009) Understanding, scoping and defining User Experience: a survey approach. Proceedings of the SIGCHI Conference on Human Factors in Computing Systems, pp. 719-728, ACM.

44. Garrett, J. J. (2002) The Elements of User Experience: User-Centered Design for the Web. Peachpit Press.

45. Rauschenberger, M., Schrepp, M., Perez-Cota, M., Olschner, S., and Thomaschewski, J. (2013) Efficient Measurement of the User Experience of Interactive Products. How to use the User Experience Questionnaire (UEQ). Example: Spanish Language Version. International Journal of Interactive Multimedia and Artificial Intelligence, Bd. 2 Special issue Artificial Intelligence and Social Aplication, 2.

46. Aizpurua, A., Harper, S., and Vigo, M. (2016) Exploring the relationship between web accessibility and user experience. International Journal of Human-Computer Studies, 91, 13-23.

47. Petrie, H. and Kheir, O. (2007) The Relationship Between Accessibility and Usability of Websites. Proceedings of the SIGCHI Conference on Human Factors in Computing Systems, New York, NY, USA, pp. 397-406, ACM.

48. W3C (2010), Web accessibility and Usability working together. http://www.w3.org/WAI/intro/usable.

49. W3C (2005), Introduction to Web Accessibility. http: //www.w3.org/standards/webdesign/accessibility.

50. ISO (2012), ISO/IEC 40500:2012 Information technology - W3C Web Content Accessibility Guidelines (WCAG) 2.0. http://goo.gl/ohwtmY.

51. W3C (2016), Techniques for WCAG 2.0. https://www. w3. org/TR/WCAG20-TECHS/G65.html.

52. Chen, Y.-H., Germain, C. A., and Rorissa, A. (2009) An analysis of formally published usability and web usability definitions. Proceedings of the American Society for Information Science and Technology, 46, 1-18.

53. Petrie, H. and Bevan, N. (2009) The evaluation of accessibility, usability and user experience. The universal access handbook, pp. 10-20.

54. ISO (2008), ISO 9241-151:2008, Ergonomics of humansystem interaction - Part 151: Guidance on World Wide Web user interfaces. http://goo.gl/9kvPYw.

55. US Department of Human Health (2006) The ResearchBased Web Design \& Usability Guidelines. Essentials of research methods in psychology.

56. Fenn, T. and Hobbs, J. (2014) The Information Architecture of Meaning Making. Reframing Information Architecture, pp. 11-30, Springer.

57. IMS (2002), IMS Access for All v3.0 Public Draft Specification. http://www.imsglobal.org/activity/ accessibility.

58. ISO (2008), Information technology - Individualized adaptability and accessibility in e-learning, education and training Part 2: "Access for all" personal needs and preferences for digital delivery. http://goo.gl/EGxfsy.

59. IEEE (2002), IEEE Standard for Learning Object Metadata. https://standards.ieee.org/findstds/ standard/1484.12.1-2002.html.

60. Orr, D., Rimini, M., and van Damme, D. (2015) Open Educational Resources: A Catalyst for Innovation. OECD Publishing. 
61. Meyer, A., Rose, D. H., and Gordon, D. (2014) Universal design for learning: Theory and practice. CAST Professional Publishing.

62. McGreal, R., Kinutha, W., and Marshall, S. (eds.) (2013) Perspectives on Open and Distance Learning: Open Educational Resources: Innovation, Research and Practice. Commonwealth of Learning and Athabasca University.

63. Wiley, D., Bliss, T., and McEwen, M. (2014) Open Educational Resources: A review of the literature. Handbook of research on educational communications and technology, pp. 781-789, Springer.

64. WebAIM (2016), Motor disabilities. http://webaim.org/ articles/motor/assistive.

65. WebAIM (2016), Visual disabilities. http://webaim.org/ articles/visual/lowvision.

66. Sanchez-Gordon, S. and Lujan-Mora, S. (2015) Accessible Blended Learning for Non-Native Speakers using MOOCs. Proceedings of the 2015 International Conference on Interactive Collaborative and Blended Learning, pp. $19-24$

67. ISO (2008), Language codes-ISO 639. http://www.iso. org/iso/home/standards/language_codes.htm.

68. IANA (2016), RFC2046 MIME Media Type Parameters. http://goo.gl/Xh17J4.

69. UNESCO (2016), ISCED: International Standard Classification of Education. http://goo.gl/kNJp39.

70. Quesenbery, W. (2014), Plain language: accessibility for information. http://goo.gl/Kk0f85.

71. Nielsen, J. (1994) Usability engineering. Elsevier.

72. Nielsen, J. (2012), How Many Test Users in a Usability Study. https://www.nngroup.com/articles/ how-many-test-users/.

73. Virzi, R. A. (1992) Refining the Test Phase of Usability Evaluation: How Many Subjects is Enough? Human Factors: The Journal of the Human Factors and Ergonomics Society, 34, 457-468.

74. Faulkner, L. (2003) Beyond the five-user assumption: Benefits of increased sample sizes in usability testing. Behavior Research Methods, Instruments, $\&$ Computers, 35, 379-383.

75. Hwang, W. and Salvendy, G. (2010) Number of people required for usability evaluation: the $10 \pm 2$ rule. Communications of the ACM, 53, 130-133.

76. Caulton, D. A. (2001) Relaxing the homogeneity assumption in usability testing. Behaviour \& Information Technology, 20, 1-7. 